Insightful Discernible Loss Because The Initial Presentation In An Exceedingly Kid With General Burkitt Cancer

\author{
Dr katov Maxim, Dr Lavrova Marina
}

N. I. Lobachevsky State University Russian

\begin{abstract}
Non-Hodgkin cancer $(\mathrm{NH})$ accounts for $100 \%$ of pediatric malignancies and is that the third commonest malignancy in childhood. We have a tendency to report a previous healthy 3-yearold boy with stage IV Burkitt cancer presenting with acute visual loss. In fundus copy his pupils were fastened at VI metric linear unit in diameter while not lightweight perception. He started medical care in line with the UKCCSG 9003 protocol. The boy didn't show any visual improvement however some slight lightweight perception despite his partial remission once Civet II. He died of neutropenia sepsis before his scheduled therapy. Finally, loss of vision could be a rare presenting sign up NH. Identification medical diagnosis $\}$ is crucial as early diagnosis and effective treatment area unit vital for survival further as for visual restoration
\end{abstract}

Key words: visual impairment, Burkitt cancer, childhood

Introduction

Non-Hodgkin cancer (NHL) accounts for $100 \%$ of pediatric malignancies and is the third most common malignancy in childhood. Central system (CNS) involvement in NHL is unusual occurring in concerning $100 \%$ of cases.[1] Ocular involvement in NHL happens as primary ocular, central nervous system cancer or isolated intraocular cancer, however optic nerve and anatomical 


\section{PHARMACEUTICAL RESEARCH}

structure illness is extremely rare. At the time of presentation, signs and symptoms depend upon the location and degree of involvement, the stage of the malignancy and also the microscopic anatomy subtype of the illness. though the head and neck region is a common web site for extra nodal involvement, acute loss of vision is a rare presenting symptom of NHL. However, it may occur in youngsters, with a median age of five years. In their majority, primary tumors of the bodily cavity sinuses gift with pain and nerves palsies.[6] The identification of bodily cavity cancer involving the optic nerves ought to additionally be enclosed in the differential identification of fulminant visual loss. Of the 9 previous cases already according, seven youngsters had bilateral visual impairment and their visual ability improved in solely 2 cases. Mean age of the according cases was eight. 1 years (range 4-16 years old) with our patient being the youngest. Differential identification \{is essential is vital is crucial\} since early diagnosis and applicable treatment area unit important factors for survival. optic tract involvement by general cancer is assumed to occur from direct invasion by neoplasm cells, that tend to infiltrate so and spinal nerves or it could develop as a complication of leptomeningeal cancertosis or orbital lymphoma.[7] though, CNS involvement in NHL is thought to be a foul prognostic issue by victimization high-dose amethopterin and intrathecal therapy, event-free survival rates have considerably augmented up to seventy fifth.[8,9]Radiotherapy even for CNS involvement is most likely no additional useful in addition to therapy. Notwithstanding, it is associate choice sometimes confined to exceptional circumstances like persistent illness once therapy, like ours. In conclusion, acute visual impairment is associate very rare presenting sign in NHL in youngsters. Neuroimaging is a useful tool in establishing the identification. Early recognition and prompt management is essential to preserve or maximize visual perform. Burkitt lymphoma is named after British surgeon Denis Burkitt, who first identified this unusual disease in 1956 among children in Africa. In Africa, Burkitt lymphoma is common in young children who also have malaria and Epstein-Barr, the virus that causes infectious mononucleosis. One mechanism may be that malaria weakens the immune system's response to Epstein-Barr, allowing it to change infected B-cells into cancerous cells. About $98 \%$ of African cases are associated with Epstein-Barr infection 


\section{PHARMACEUTICAL RESEARCH}

\section{Discussion}

This report describes a patient with general Burkitt cancer illustrating associate vital purpose that loss of vision is also the presenting symptom in general NHL at identification. Previous reports have documented optic pathology with CNS involvement in adult patients with general cancer in clinical remission.[3] though series of pediatric ocular and orbital lymphomas have been according, optic nerve infiltration from general NHL within the pediatric people at identification area unit very restricted.[4] optic tract involvement has been related to lymphomatous infiltration, infections, vincristine-related pathology, toxin medication, radio necrosis and paraneoplasia.[5] Nasal-par nasal cavity cancer affects senior people most normally.

\section{References}

1. Sand Lund Jt, landscaper Junior, Crist WM. Non-Hodgkin's cancer in childhood.

2. Umami N, Daniel SJ. Acute bilateral visual impairment as a presenting symptom of NonHodgkin's cancer.

3. Lee IC, Howe's el, Bhisitkul Rib. General non-Hodgkin's cancer with optic tract infiltration in an exceedingly patient with AIDS. Tissue layer 2002.

4. Zama AG, Graham end, Sanders Md. Anterior sensory system involvement in Hodgkin's cancer. $\mathrm{Br} J$ Ophthalmic.

5. Akashi S, Sidhu t. pediatric orbital and ocular lymphomas. Pediatric Blood Cancer.

6. Evans IS, American Revolutionary leader biological warfare. Non-Hodgkin cancer. 


\section{THE AMERICAN JOURNAL OF}

MEDICAL SCIENCES AND

VOLUME01 ISSUE03

\section{PHARMACEUTICAL RESEARCH}

7. Chamberlain megacycle per second. Current ideas in leptomeningeal metastasis. Oncol. 